\title{
Point-based Medialness for Movement Computing
}

\author{
Frederic Fol Leymarie \\ Goldsmiths College \\ University of London \\ New Cross, London, U.K. \\ ffl@gold.ac.uk
}

\author{
Prashant Aparajeya \\ Goldsmiths College \\ University of London \\ New Cross, London, U.K. \\ p.aparajeya@gold.ac.uk
}

\author{
Carol MacGillivray \\ Goldsmiths College \\ University of London \\ New Cross, London, U.K. \\ c.macgillivray@gold.ac.uk
}

\begin{abstract}
We introduce the idea of using a perception-based medial point description of a biological form (such as a 2D profile of a moving animal) as a basis for movement computing which delivers computational schemes to automatically annotate movement and be capable of producing meaningful qualitative descriptions. We distinguish interior from exterior shape representation. Interior medialness is used to characterise deformations from straightness, corners and necks, while exterior medialness identifies the main concavities and inlands which are useful to verify parts extent and reason about articulation and movement. We define an interior dominant point as a well localised peak value in medialness representation, while an exterior dominant point is evaluated by identifying a region of concavity sub-tended by a minimum angular support. Furthermore, significant convex points are extracted from the object's form to further characterise the elongation of parts. We propose that our evaluated feature points are sufficiently representative, as a basis for shape characterisation, to address many of the goals of movement computing.
\end{abstract}

\section{Categories and Subject Descriptors}

I.4.7 [IMAGE PROCESSING AND COMPUTER VISION]: Feature Measurement —representation, shape

\section{Keywords}

Medialness. Line of Action. Codons. Visualising movement.

\section{INTRODUCTION}

In this short communication we introduce our proposed shape representation for movement computing applicable to animated biological objects 11 which is inspired by results and techniques from cognitive psychology, artistic rendering and animation and computer vision. Figure 1 (top row) shows an artistic way to draw specific movements of an animal where the artist perceives a biological

\footnotetext{
${ }^{1}$ We present results for human-scale animals, but the method also can be applied to smaller animals, like insects, or plants (bending, deforming), and artistic objects like animated characters.

Permission to make digital or hard copies of all or part of this work for personal or classroom use is granted without fee provided that copies are not made or distributed for profit or commercial advantage and that copies bear this notice and the full citation on the first page. Copyrights for components of this work owned by others than the author(s) must be honored. Abstracting with credit is permitted. To copy otherwise, or republish, to post on servers or to redistribute to lists, requires prior specific permission and/or a fee. Request permissions from Permissions@acm.org.

MOCO'14, June 16-17 2014, Paris, France.

Copyright is held by the owner/author(s). Publication rights licensed to ACM. ACM 978-1-4503-2814-2/14/06 ...\$15.00.

http://dx.doi.org/10.1145/2617995.2618001.
}

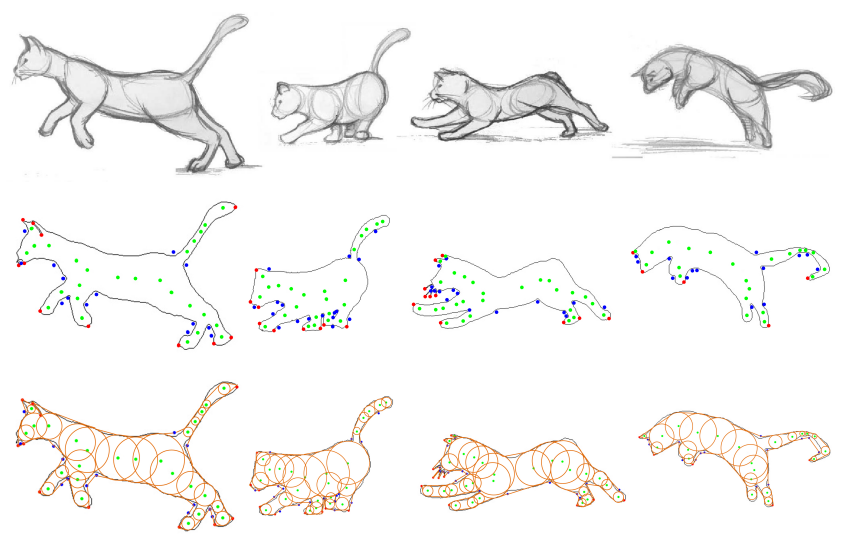

Figure 1: Top row: An artistic way to draw animal forms, here of a playful cat (Artist: C Kelvin Chow). Middle row: Our proposed shape representation in terms of dominant medial (in green) and contour (convex (red) and concave (blue)) points. Bottom row: A possible set of contour reconstructions of the moving cat using our proposed point-based medialness representation.

character as the combination of primitive structures (here approximate disks of various radii). Each body movement is characterised by a particular combination in size and orientation of these primitives. From the point of view of psychophysical investigations on the perception of shape movements by humans, Kovács et al. have shown that such articulated motions can best be captured via a minimal set of dominant features, potentially being represented as isolated points [18]. Inspired with these two approaches to the perception of natural motions, we have investigated a possible computational scheme based on the notion of robust medialness presented by Kovács et al. that can capture the important structural part-based information commonly used in artistic drawings and animations. The main advantage over other classical medial-based representations of shape is one of combined compactness, robustness and capacity of dealing with articulated movements. Note also that we do not require to have a complete object segmented and thus can produce movement computing descriptions from partial object views (e.g. under occlusions).

Shape representation has been addressed in many ways by computer scientists, including: by contour analysis [7], using Blum's medial axis transform (MAT) and its related 2D shock graph [16 28], combining contour and a skeletal medial graph [21, 1], or using instead the related inner distance [23]. Closest to our approach from a computational point of view are the contour enclosure-based 
symmetries [15] and medial point transform [31] - but in these earlier works no attempt is made to isolate and use dominant points. Other classical approaches emphasise similarly either boundary information (e.g. Fourier, wavelet and scale-space analyses of closed contours) or interior information (e.g. primitive retro-fitting or approximation). Most of these (medial or not) shape-based approaches do not explicitly tackle deformations and articulated movements [24], while we do.

Our representation, derived from the earlier proposal by Kovács et al. [18], maps the whole shape information into a few number of points we call "dominant". Contrarily to classical medial-based representations, ours is not overly sensitive to small boundary deformations and furthermore gives high response in those regions where the object has high curvature with large boundary support and in the vicinity of joints (between well-delineated parts, such as the limbs of an animal). We augment the medial dominant points with main contour points indicating significant convex and concave features, thus bringing together with our notion of medialness the main $2 \mathrm{D}$ point-based shape systems proposed over the years in the fields of cognitive psychology and computer vision: the so-called "codons" denoting contour parts [27] and high curvature convexities often used in scale-space analyses [24].

\section{MOTIVATIONS \& BACKGROUND}

By movement computing we understand a developing discipline which aims at providing computational schemes to automatically annotate movement and be capable of producing meaningful qualitative dynamics descriptions, including the inherent quality of a movement, its phrasing, embodiment and motivation, which are integral and should be included as parameters [8, 20].

The earliest known attempts to annotate movement were in dance and date from the 15th century [12], but the first true annotator of movement to include a strong qualitative element was Rudolf Laban who created a Labanotation in the first half of the 20th Century, based on the idea of harmonic movement [10, p.77]. Originally designed for choreography and dance, Labanotation has developed into one of the most widely used systems of human movement analysis in dancing, in Jungian therapies such as authentic movement [26], and as a tool for analysing the movement of actors [25]. Laban created scales (similar to musical scales) in imaginary structures made of platonic solids or "kinespheres". His notation splits movement into two components: kinematic, the quantitative position of a body or its parts in space at one time, and the non-kinematic (harmonic) components of Effort and Shape, where relative qualities of expressive force are annotated [8, 20].

Prior to Laban, two important pioneers of camera systems to record and study movement were the late 19th century chronophotographers Eadweard J. Muybridge and Étienne-Jules Marey. Both took a keen interest in uncovering the details of movement in people and animals by filming motions in a series of sequenced stills. Muybridge's system used multiple cameras that shot stills spatially separated, and the physical movement in space tripped a photograph being taken. Marey further developed this idea by designing different cameras, including what is to be considered the first modern film camera replacing glass plates with sensitised paper and later transparent celluloid film. Marey also was the first to introduce the concept of tracking light dots over a dark suit and background, where the dots are positioned along a skeletal frame to study in particular the movement of humans, a technique reinvented in the 1970's and modernised today in motion capture systems [17]. Muybridge and Marey proved many new facts about movement such as studying the way a horse galloped and if its four hooves ever completely left the ground in the process (they do). They also made numerous studies of everyday movements of people [5]. With the advent of cinematic film, studies of natural movement by stills was left to anthropologists recording ethnic interactions and to the early stages of animation, where senior animators drew extreme "key-frames" of action with frame timings leaving the "in-between" drawings to be carried out by less experienced animators. Even today 3D animators in games and films create key-frames, but now the in-betweening is made with software. Lately there has been a rise of interest in the notion of key-framing for notating movement as it cuts out extra data [14, 13].

Animation takes motivation as a central tenet. Motion in animation cannot be discussed without referring to the twelve animation principles first espoused by the Disney studios in the 1930's and still going strong today [29]: 1- Squash and Stretch; 2- Timing; 3- Anticipation; 4- Staging; 5- Follow Through \& Overlapping Action; 6- Straight Ahead \& Pose-To-Pose Actions; 7- Slow In(to) and Out; 8- Arcs; 9- Exaggeration; 10- Secondary Action; 11- Appeal; and 12- Personality. The fact that these principles have stood the test of time and have been adapted successfully to new methods of animating such as in 3D Computer Graphics [19] means that they underpin most animator's work today every bit as much as they did on Walt Disney's first animated feature film "Snow White and the Seven Dwarfs" a lifetime ago (in 1937).

With all these principles in their toolkit the animator has no problem communicating and an animated version would certainly include full body movement and motivation. It has been pointed out by Leslie Bishko that there is some similarity between Labanotation and these principles of animation in expressing the functional aspects of movement [3]. While animation principles permit to impose a specific style of movement (such as in cartoons) they lack the depth and richness of Labanotation in exposing not only functional aspects of movement but their detailed expression [4]. However, animation principles do not yet form the basis of a theory which can lead to formal models, ease of simulation and computational implementations [9]. Similarly, Labanotation lacks complete computational models, as the representation and analysis of qualia in the notions of Effort and Shape remain very challenging, but the theory is more developed and progress is being made [8, 30].

One important tool used in animation for controlling qualia which offers the potential for implementation is the idea of a central line of deformation being key to our assimilation of full body movement. The Line of Action (LoA) is a single line running through the character, which represents the overall force and direction of each drawing. In traditional celluloid animation such as used by early Disney full-length features like Snow White, before drawing the full character, an animator would frequently draw in a LoA to help determine the position of the character. By simply changing the LoA - making it more curved, sloped or arched in a different direction - the entire essence of the drawing can be changed. Bregler et al. have investigated capturing such a contour (the LoA) as the source of the motion and re-targeting it to other characters in other media. Although there is not enough information in this contour to solve for more complex motion, such as how the legs move relative to each other, the investigators discovered that a surprising amount of information comes from this single curve: the essence of the motion is still present in the re-targeted output [6]. A more recent application of the LoA to $3 \mathrm{D}$ character interactive animation is being explored at the INRIA in France [11].

The proposed representation via medial nodes of Kovács et al. can be seen as a potential psychologically motivated support for the LoA, where the later is a simplification of a full medial trace. Kovács et al. define a pseudo-distance function, $D_{\epsilon}$, which captures medial symmetries within a pair of disks defining an annulus 


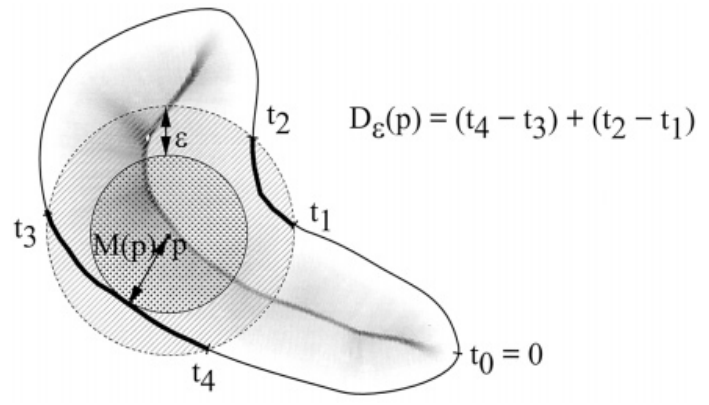

Figure 2: Adapted from [18]. The $D_{\epsilon}$ function for a simple shape defined as a sum of boundary segments falling inside the annulus neighbourhood of thickness $\epsilon$ (thick boundary segments within the grey ring) centred around the circle (with centre $p$ ). $M(p)$ is taken as the minimum radial distance from point $p$ to the nearest contour point.

region, where " $\epsilon$ " denotes the (parametric) thickness of the annulus [18 17]. Under this metric, the special nodes of the skeletal field are those which locally maximise the amount of outline trace they capture: they are "the most informative points along the skeleton" of an object in motion. Such a compact medial representation shares strong resemblance to the chronophotographs of Marey (circa 1880) and to G. Johansson's work (circa 1970) on the perception of biological motion for point-light walker displays [17]. The $D_{\epsilon}$ distance function of Kovács et al. can be computed for each frame of a video sequence, when applied directly to available outlines. "The maxima of the function are good candidates as primitives for biological motion computations" [18, 17]. Such a representation of bodies in movement by a graph connecting nodes of high (visual) interest is a potentially richer model than the single LoA used in (cartoon) animation. It also offers an explicit markerless computational model and relates to human perception.

\section{COMPUTATIONS}

Mathematically, following Kovács et al. [18], medialness of a point in the image space is defined as the containment of the longest segment of boundary (information, edges) falling into the annulus of thickness parametrised by the tolerance value $(\epsilon)$ and with interior radius taken as the minimum radial distance of a point from boundary (Figure 2). On completion of medialness measurements each pixel in the transformed image space holds a local shape information (of accumulated medialness). Assuming figure-ground separation, thickness variations, bulges and necks of an object are captured via interior medialness measurement, while the concavities and joints are defined via exterior medialness measurement. The interior and exterior medialness measurements give different types of information about the shape of an object and they are processed separately in our proposed method.

In the work of Kovács et al. it is shown that humans are most sensitive to a small number of localised areas of medialness [18]. Our equivalent (extended) notion is defined as dominant points and can be applied to any objects, animated or not. Dominant points are constrained to be a relatively small number of points of high medialness obtained by filtering out the less informative, redundant and noisy data from the initial medialness image space.

To identify internal dominant points a top-hat transform [32] is applied to isolate peaks in the medialness signal. Peaks are filtered using an empirically derived threshold. The selected peaks are then each characterised by a single representative point. To avoid con- sidering large numbers of nearby isolated peaks which are characteristic of object regions with many small deformations, only peaks at a given minimum distance away from each other are retained. The extraction process of external dominant point is achieved by combining a concavity measure together with length of support on the contour. Again, a spatially localised filtering is applied to isolate representative dominant points. Furthermore, to improve the robustness of our representation, we extract a set of convex points to capture the protrusion-like structure of an object. All the selected internal and external dominant points along with convex points are then considered as the representative feature points of the shape. This computational scheme can then be applied to a set of frames in a movement sequence.

\subsection{Medialness Measure}

A medial point is defined by computing the $D_{\epsilon}$ function based on an equidistant metric (to boundary segments). The $D_{\epsilon}$ value at any point in space represents the degree to which this point is associated with a percentage of bounding contour pixels of the object within a tolerance of value $\epsilon$ (after Kovács et al. [18]; Figure

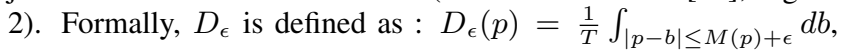
for any point $p=\left[x_{p}, y_{p}\right]$, vector $b(t)=[(x(t), y(t)]$ describing the $2 \mathrm{D}$ bounding contour $(B)$ of the object, and normalising factor $T=\int_{b \in B} d b$. The metric $M(p)$ is taken as the smallest distance between $p$ and the bounding contour: $M(p)=\min _{0 \leq t \leq 1}|p-b(t)|$. In Figure 6 (top row), the medialness measurement is performed on a standing dog showing the variation in internal medialness measurement (different value of the $D_{\epsilon}$-function) by augmenting the value of tolerance $(\epsilon)$ which reflects a smoothing effect: as $\epsilon$ increases, smaller symmetries are discarded in favour of large scale ones (external measurements are described later).

\subsection{Dominant Points Extraction}

Medialness measurement is currently done separately for internal and external regions, taking advantage of the perceptual figureground dichotomy known to be a powerful cue in humans. This also enables our method to consider articulated objects as potential targets in pattern recognition tasks, as will become clearer later. Each point in the transformed image space has some local shape information in the form of medialness for a globally selected tolerance $\epsilon$. Among these points, some have, cumulatively, enough information to represent parts or ultimately the whole object. Consequently, our hypothesis is that by using Kovács et al.'s definition of medialness the entire shape of a 2D object can be confined into a few number of salient points, which we refer to as dominant.

\subsubsection{Internal Dominant Points}

Medialness increases with "whiteness" in our transformed images (which proves also useful for its visualisation). To select points of internal dominance, a "white" top-hat transform is applied, resulting in a series of bright white areas. The white top-hat transform is defined as the difference of an input function (here an image of medialness measures as a grey-level 2D function) with the morphological opening of this function by a flat structural element (a disk parametrised by its radius as a function of $\epsilon$ ). Opening is a set operator on functions which "removes" small objects from the foreground of an image, placing them in the background (augmenting the local function set values) [32]. This filtering is followed by a thresholding to discard remaining areas of relatively low medialness significance. Figure 3 b) shows the result obtained after applying the white top-hat transform on a medialness image 3 a).

We still require to process further the output of the top-hat transform to isolate the most dominant points amongst the remaining 

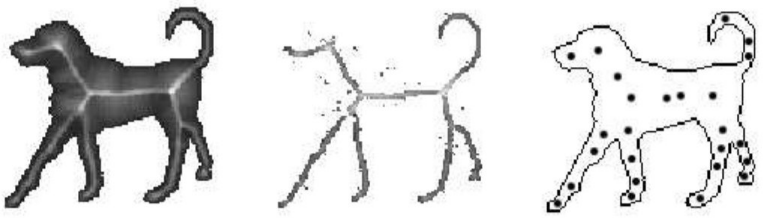

Figure 3: The 3 successive steps in isolating internal dominant points: (i) medialness representation of (the interior of) a dog figure; (ii) top-hat transform; and (iii) internal dominant points shown as enlarged black dots.

selected medialness points which tend to form clusters. To do so, a flat circular structuring element of radius $\epsilon / 2$ (but of at least 2 pixels in width) is applied over the top-hat image - such that within the scope of the structural element, only that locus which maximises medialness is selected.We further impose that no remaining points of locally maximised medialness are too close; this is currently implemented by imposing a minimum distance of length $2 * \epsilon$ is taken between any pair of selected points. We have found that in practice this is sufficient to avoid clustering interior dominant points (an example of the result of this filtering is shown in Figure 3 (c)).

\subsubsection{External Dominant Points}

In practice, if an object can be deformed or is articulated, salient concavities can be identified in association to those deforming or moving areas (such as for joints and limbs of a robot or human body). Considering this empirical observation, the location of an external dominant point can be made invariant to this deformation/articulation only up to a certain extent. For example, if the location of an external dominant point is initially relatively far away from the corresponding contour segment, a slight change in the boundary shape near the movable part (such as an arm movement) can considerably change the position of that associated dominant point (Figure 4 left). On the other hand, if a point is located very close to the contour, it can easily be due to noise or small perturbations of the boundary.
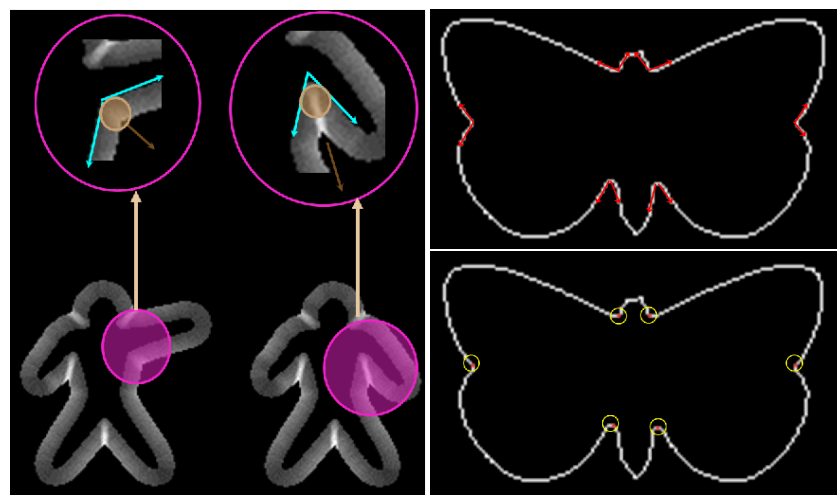

Figure 4: Left: External medialness processing on a humanoid. The articulated movement of the left arm changes the location and orientation of the associated external dominant point. If located far from the contour, it then proves difficult to retrieve a (shape-based) match with the modified form. Blue arrows show the local support for concavity while brown arrows indicate the direction of flow of medialness (away from the concavity). Right: Top: Detection of concave regions (on a butterfly) using angular support. Bottom: Detected Concave points.
In practice, to resolve these issues, we define a candidate external dominant point as a local concavity if it falls under a threshold angular region, with a constraint of length of support which itself depends on the tolerance value $(\epsilon)$. The value of the threshold $\left(\theta_{\text {out }}\right)$ is tunable but is always less than $\pi$, which permits to control the angular limit of the concave region. A locus whose concavity is larger than $\theta_{\text {out }}$ is considered a flat point. In our experiments we tuned the value of $\theta_{\text {out }}$ from $5 \pi / 6$ to $8 \pi / 9$. In association, we define an external circular region (of radius function of $\epsilon$ ) centred at each concavity containing candidate external dominant points. Each such region may provide only one representative dominant point, as a function of the maximum containment of boundary points inside the associated annulus (of medialness) and corresponds to (our definition of) the maximum length of support. Finally, we position the representative dominant point to be near and outside the contour at a fixed distance (Figure 4, right).

\subsubsection{Convex Points}

Our final shape feature is a set of convex points, where an object has a sharp local internal bending and gives a signature of a blob-like part or significant internal curvature structure (i.e. a peak in curvature with large boundary support). The goal is to represent an entire protruding sub-structure using one or few boundary points. Such protrusions are known to be important contributors in characterising shape [27, 21, 2]. The process of extraction of convex points is very similar to the extraction of concave loci, one difference being in the value of the threshold angle $\left(\theta_{i n}\right)$, where $\pi<\theta_{\text {in }} \leq 2 \pi$. In our experiments we have found useful values to be in the range: $5 \pi / 4$ to $4 \pi / 3$.

Such convex and concave points are complementary to each others and have been used in the "codon" theory of shape description: a codon is delimited by a pair of negative curvature extrema denoting concavities and a middle representative positive maximum of curvature denoting a convexity [27]. In our case we associate these two sets with the extremities of the medial axis of H. Blum: end points of interior branches correspond to centre of positive extrema of curvature and end points of exterior branches are mapped to negative extrema of curvature of the boundary. The repositioning of these extrema near the boundary is alike the end points of the PISA (Process Inferring Symmetry Axis) representation of M. Leyton [22]. Together, the three sets: concave, convex and interior dominant, form a rich enough point-based description of medialness to allow us to efficiently address applications with articulated movement for real image data.

\subsection{Articulation}

Anatomically, an animal's articulated movement is dependent on the point of connection between two bones or elements of a skeleton. Our results show that the concave points (representative exterior dominant points) have good potential to indicate and trace such articulations, unless the shape is highly deformed. For usual movements (e.g. walking, jogging, gesticulating), these feature points remain present and identifiable in association to an underlying bone junction and hence can provide a practical signature for it; examples of this property are given in Figures 5,6 and 7

\section{DISCUSSION}

We have presented early results in applying our proposed pointbased medialness representation to support movement computing. Medialness here refers first to the model proposed by Kovács et al. in terms of "hot spots" along the thick trace of medial measures for the interior of a biological form (in movement or in a static pose) [17]. We introduced here a possible algorithmic method to 

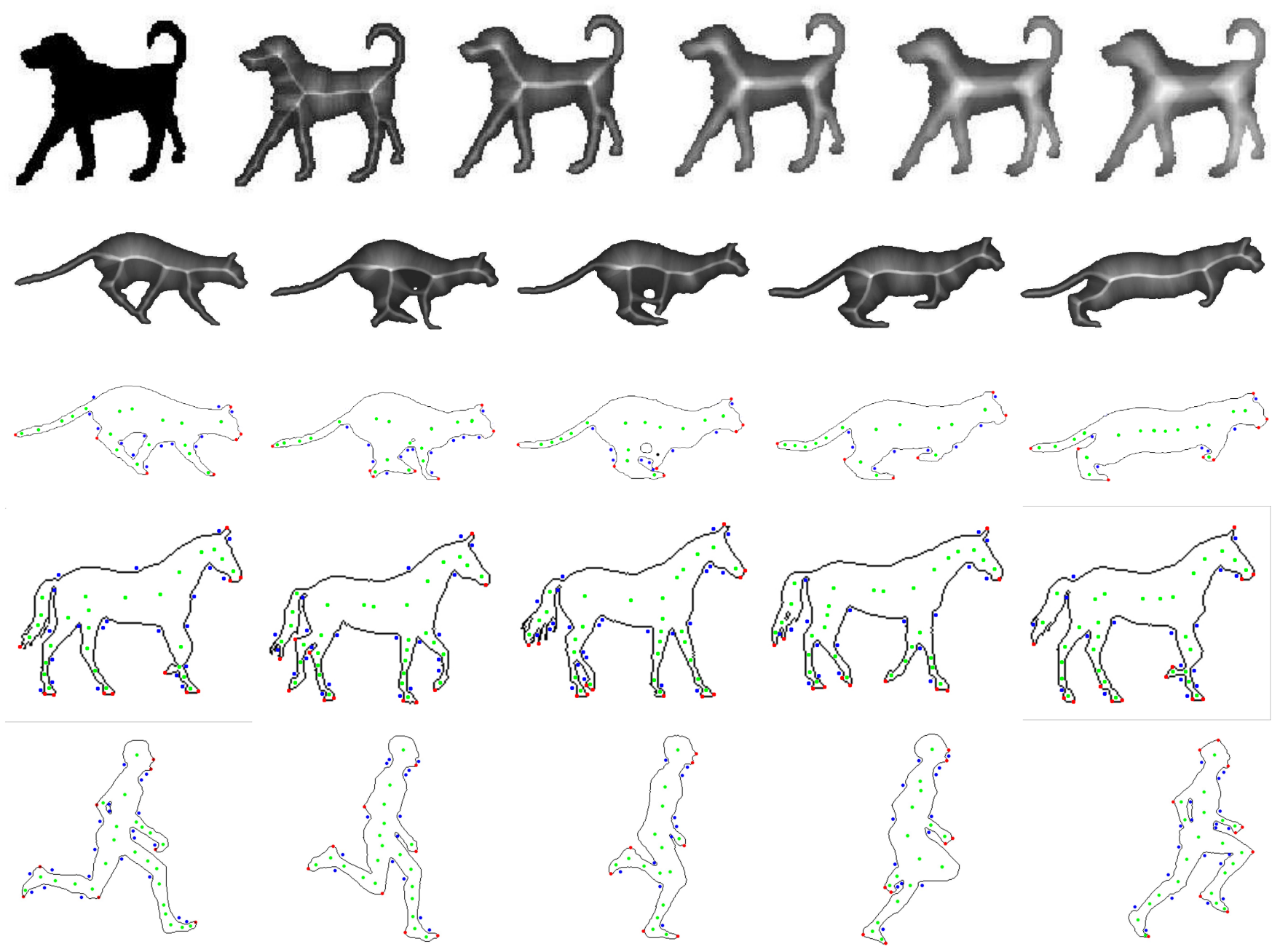

Figure 6: Top row: Medialness and tolerance. Left: A dog taking a pose. Other images to the right show variations in $D_{\epsilon}$ for increasing tolerance values $(\epsilon=2,4,8,12,16)$. 2nd row: The $D_{\epsilon}$-function for a sequential set of frames of the movement of a running cat. The maxima (white spots) of the function are good candidates as primitives for biological motion representation. $3 r d$ $\&$ 4th rows: Illustration of the changes in feature points loci for the (same) running cat and a trotting horse. Bottom row: Similar illustration for a few frames of a running athlete captured by E. Muybridge in 1887.
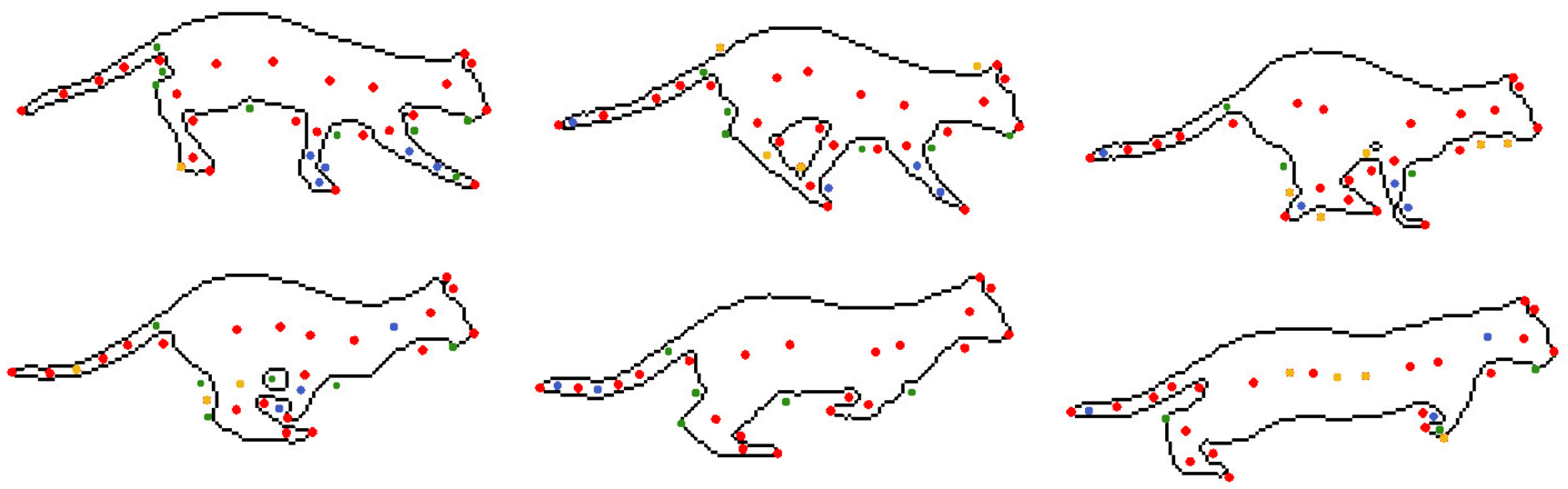

Figure 7: A running cat captured in 6 different frames. Different dominant points (internal dominant, external dominant and convex points) are shown using colours to indicate their persistence in time: Red: present in all frames; Green: highly frequent (4-5 times); Blue: less frequent (2-3 times), and Yellow: not consistent (single occurrence). NB: Due to the animal's movement, sometimes dominant points overlap each other. 

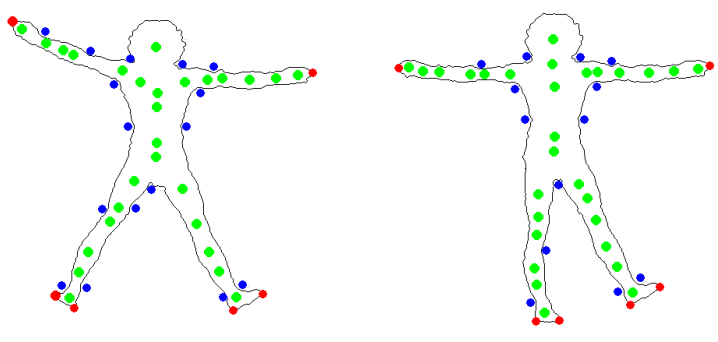

Figure 5: Two samples from an articulated set of Vitruvian men (after L. da Vinci, 1490) illustrating some features of our perception-based selection of dominant points.

extract such hot spots as dominant points of interior medialness. We extend this representation by augmenting it with notions of concave and convex points located near the outline and based on local medialness measures. Note that we have applied Kovács et al. medialness measure also to the exterior of a form, in order to characterise concavity (a further extension of their model) and to capture a part-based representation of shape (combining it with the codon and PISA models). Note also that all our parameters (e.g. in morphological filtering) are set with respect to the tolerance level in medialness, $\epsilon$, which is currently globally set to a single value. One area of future experimentation will be to evaluate the use of multi-scale medialness (e.g. refer to Fig 6 (top row)) versus a locally varying $\epsilon$ (alike adaptive/anisotropic smoothing).

Beyond perception-based models, we also are trying to capture useful concepts from animation and more generally the art of drawing forms in movement. The Line of Action which can be interpreted both as a simplification of medialness (a single medial curve) and an extension (applicable to more than one form at a time) can potentially be represented on the basis of our medial point set by selecting and interpolating a subset. Such an interpolation can also be used to recover a skeletal graph or sketch which can feed in recently proposed information retrieval methods applied to video archives of dance footage [14, 13]. We are also exploring the relation between drawing techniques using primitives (like disks) to create a scaffolding for a final drawn rendering of a moving form (Fig. 1).

One of our motivations for wanting to relate to drawing and animation is to combine qualitative with quantitative descriptions of movement. This has lead us to also consider the field of dance notation where a rich tradition of bridging such a gap exist. Currently, we are exploring the notation of Eshkol-Wachman which is built from an explicit skeletal graph [20].

Our work also directly relates to the early findings of Marey and Muybridge. Ours can be seen as providing a marker-less approximation to solve the inherent problem of capturing space and time information in one notation from an analysis based on photographic snapshot sequences or video (Fig. 6 bottom 3 rows). We are still exploring the full potential of this representation; e.g. in Fig. 7 we track our feature point set over a frame sequence to highlight their dynamics (here persistence in time).

\section{ACKNOWLEDGEMENTS}

This work was partially funded by the European Union (FP7 ICT - FET; Grant Agreement \#258749; CEEDs project). We thank the artist Kelvin Chow for his drawings (Fig. 1 top row)).

\section{REFERENCES}

[1] X. Bai, W. Liu, and Z. Tu. Integrating contour \& skeleton for shape classification. In ICCV Workshops, pages 360-7, 2009.

[2] S. Berretti et al. Retrieval by shape similarity. IEEE Trans. on Multimedia, 2(4):225-39, 2000.

[3] L. Bishko. Relationships between LMA \& computer animation. In Dance \& Technology 1. Fullhouse, 1993.

[4] L. Bishko. The uses and abuses of cartoon style in animation. Animation Studies Online Journal, 2:24-35, 2007.

[5] M. Braun. Picturing Time: The Work of Etienne-Jules Marey. University of Chicago Press, 1995.

[6] C. Bregler et al. Turning to the masters: Motion capturing cartoons. ACM Trans. on Graphics, 21(3):399-407, 2002.

[7] L. Chen et al. Efficient partial shape matching using Smith-Waterman algorithm. 2008. CVPR Wksh., pp. 1-6.

[8] D. Chi et al. The EMOTE model for effort and shape. In Proc. of SIGGRAPH, pages 173-82, 2000.

[9] M. S. El-Nasr et al. Believable characters. In Hdbk Multimedia f/Digi. Ent. \& Arts, pages 497-528. Springer, 2009.

[10] S. L. Foster. Reading Dancing. U. of Calif. Press, 1986.

[11] M. Guay et al. The Line of Action: An intuitive interface. ACM Trans. on Graphics, 32(6):Article no. 205, 2013.

[12] A. Hutchinson Guest. Choreographics. Routledge, 1998.

[13] S. James et al. ReEnact: Sketch based choreog. design from archival dance footage. In ACM - ICMR, pp. 313-20, 2014.

[14] M. Kapadia et al. Efficient motion retrieval in large motion databases. In ACM Proc. of i3D, pages 19-28, 2013.

[15] M. Kelly and M. D. Levine. Annular symmetry operators. In ICCV, pages 1016-21, 1995.

[16] B. Kimia. On the role of medial geometry in human vision. Journal of Physiology - Paris, 97(2):155-90, 2003.

[17] I. Kovács. "Hot spots" and dynamic coordination. In Dynamic Coord. in the Brain. MIT Press, 2010. pp. 215-28.

[18] I. Kovács, Á. Fehér, and B. Julesz. Medial-point description of shape. Vision research, 38(15):2323-33, 1998.

[19] J. Lasseter. Principles of traditional animation applied to 3D comp. anim. Computer Graphics, 21(4):35-44, 1987.

[20] N. Lehoux. Dance literacy and digital media. Int'l J. of Performance Arts \& Digital Media, 9(1):153-74, 2013.

[21] F. Leymarie and M. D. Levine. Simulating the grassfire transform. IEEE PAMI, 14(1):56-75, 1992.

[22] M. Leyton. A process-grammar for shape. Artificial Intelligence, 34(2):213-247, 1988.

[23] H. Ling and D. Jacobs. Shape classification using the inner-distance. IEEE PAMI, 29(2):286-99, 2007.

[24] Y. Mingqiang et al. A survey of shape feature extract. tech. In Pattern Rec. Tech. \& Applic. pp. 43-90, InTech, 2008.

[25] J. Newlove. Laban for Actors \& Dancers. Nick Hern, 1993.

[26] P. Pallaro ed. Authentic Movement. Jess. Kingsley, 1999.

[27] W. Richards and D. D. Hoffman. Codon constraints on closed 2D shapes. CVGIP, 31(3):265-81, 1985.

[28] T. Sebastian et al. Recognition of shapes by editing their shock graphs. IEEE PAMI, 26(5):550-71, 2004.

[29] F. Thomas and O. Johnston. Disney Animation. 1981.

[30] L. Torresani et al. Learning motion style synthesis from perceptual observations. In NIPS, pages 1393-1400, 2007.

[31] G. van Tonder and Y. Ejima. Flexible computation of shape symmetries. IEEE SMC, Part B, 33(3):535-40, 2003.

[32] L. Vincent. Morphological grayscale reconstruction in image analysis. IEEE Trans. Ima. Process., 2(2):176-201, 1993. 